\title{
Rituales de interacción sexual entre hombres. Una propuesta de análisis del discurso y de la práctica del sexo anónimo
}

Rituals of sexual interaction between men. A proposal for the analysis of discourse and practice of anonymous sex

José Antonio Langarita Adiego

Profesor Visitante. Departamento de Pedagogía. Universitat de Girona (España)

josan.langarita@udg.edu

CUERPOS, SEXUALIDADES YPODER

MONOGRÁFICO COORDINADO POR JOSÉ MARÍA VALCUENDE. Universidad Pablo de Olavide (Sevilla).

\section{RESUMEN}

En este artículo se presenta un análisis de la práctica del sexo anónimo entre hombres. El objetivo es demostrar que se trata de una actividad que responde a una realidad social hostil hacia aquellas prácticas alternas a la heterosexualidad y la monogamia. La obtención de los datos presentados se ha realizado fundamentalmente a partir de la observación participante y la entrevista, aunque la revisión de registros literarios también ha contribuido a la redacción definitiva del artículo. Para ello se propone un análisis del sexo anónimo entre hombres desde la perspectiva de los rituales de interacción sexual, donde existen unas normas y ordenes que cada participante debe conocer y reproducir para conseguir los objetivos del encuentro. Este punto de vista nos permite entender y reconocer el proceso de negociación y acceso al sexo a partir del principio de anonimato.

\section{ABSTRACT}

In this study, I analyse anonymous sex practices between men. My aim is to demonstrate how this activity is a response to the hostile social reality towards those who practice alternatives to acts of heterosexuality and monogamy. To gather the data presented, I used participant observation and interview as my main technique, although the review of literature has also aided in the construction of the final proposal. To achieve this proposal, I perform an analysis of anonymous sex between men, viewed from the perspective as being a sexual interaction ritual with rules and orders that each participant must know and adhere to in order to reach the goal of the encounter. This viewpoint allows us to understand and recognize the process of negotiation and access to the sex from the principle of anonymity.

anonymous sex| interaction rituals | homosexuality | GLTB studies | Anthropology of sexuality

\section{Introducción}

La práctica del sexo anónimo entre hombres en espacios públicos se conoce hoy en día con el anglicismo cruising. En otros momentos de la historia reciente de España se ha llamado a esta actividad hacer la carrera, que es precisamente el nombre que utilizan las prostitutas para referirse a su trabajo en la calle (Guasch 1991). Sin embargo, la influencia anglosajona en el entorno homosexual de las últimas décadas ha hecho que quienes participan en esta actividad se apropien del concepto inglés para referirse al intercambio sexual anónimo en parques, playas, aparcamientos de coches, lavabos u otros emplazamientos públicos.

En este trabajo se presta atención exclusivamente a las prácticas de sexo anónimo entre hombres en espacios públicos. Aunque existen otros entornos de sexo anónimo heterosexual, como el cancaneo o doging, y homosexual como saunas o cuartos oscuros, estos no forman parte del análisis que se propone en este artículo. Cabe señalar, no obstante, que las lesbianas aseguran que no existen lugares con estas características para encuentros sexuales anónimos entre mujeres.

En las siguientes páginas se tratará de discutir las repercusiones de las narrativas y discursos entorno al sexo anónimo entre hombres y, a su vez, presentar las estrategias de los usuarios de las zonas de cruising 
para salvaguardar esta forma particular de acceso al gozo sexual. Para ello, este artículo se apoya en un análisis de la práctica desde la perspectiva ritual, de manera que se pueda profundizar en cómo la ritualización que conduce los contactos entre hombres da acceso al placer sexual preservando la identidad y la identificación de los participantes.

El trabajo de investigación que se presenta se ha llevado a cabo a partir de una aproximación cualitativa de carácter etnográfico en la que la observación participante y la entrevista han sido las técnicas fundamentales para el acceso a la información. La observación participante ha jugado un papel clave, ya que ha permitido al investigador experimentar e intervenir en los procesos de acceso, negociación y acto sexual de las zonas de cruising. Por otro lado, las entrevistas y conversaciones informales con usuarios, entidades y administración pública le han llevado a comprender algunas de las perspectivas y discursos de los diferentes actores sociales respecto a la práctica del cruising, así como a conocer las motivaciones de los participantes para inmiscuirse en esta actividad. No obstante, la revisión bibliográfica ha sido especialmente relevante para construir las narrativas institucionales, literarias y de otras épocas.

En cuanto al escenario de investigación, se centra en lugares al aire libre: el parque de Montjuïc de Barcelona, la playa de Gavà y el bosque y la playa de Sitges han sido los espacios que han nutrido buena parte de los datos que aquí se presentan, ya que fue allí donde se realizó el trabajo de campo entre los años 2009 y 2013.

\section{El contexto}

Algunos historiadores afirman que en el siglo XVII ya existían diferentes lugares de intercambio sexual entre hombres en espacios públicos (Aldrich 2004: 1724, Hahn 1979: 13, Sibalis 1999 y Vázquez 2010: 268). Pero es necesario destacar que la práctica del sexo anónimo entre hombres no se desarrolló de manera aislada y sin contexto social, sino que requirió de unas condiciones urbanas y sociales que le permitiesen construir un espacio social propio. En el siglo XVIII una nueva forma de vida pública había cobrado una gran relevancia gracias a la modernización de la ciudad que se construía bajo el prisma de una burguesía en ascenso y un crecimiento acelerado de población que permitía que el encuentro entre extraños fuese algo probable (Sennet 1977: 68). Caminar por las calles, ver y ser visto se había convertido en una actividad social del siglo XVIII. Además, el cuerpo había tomado una nueva interpretación social en la que se admitía y reconocía la necesidad de la intimidad corporal. La higiene y limpieza de los excrementos pasó a ser una práctica específicamente urbana y de clase media lo que provocó que, junto con la nueva concepción del cuerpo y de la presencia pública se transformasen también, en buena medida, las ciudades y las prácticas que se daban en ellas (Sennet 1994: 281-282). Ahora bien, la posibilidad de expulsar las deyecciones humanas de la vida pública y la concepción de la pulcritud renovada se pudo llevar a cabo únicamente porque se crearon los utensilios técnicos que lo permitieron (Elías 1977: 181), es decir, los urinarios públicos y el sistema de desagüe. Los urinarios públicos poco a poco se instalaron como recursos urbanos que permitían a las personas excretar en lugares propios y destinados para ello. Sumado a esto, se construyeron los parques de las ciudades como espacios en los que los ciudadanos podían salir a relajarse del tormento de las calles y tomar el aire fresco (Sennet 1994). El parque se convirtió en un nuevo lugar social donde los sujetos miraban y eran mirados, un lugar asociado al descanso, desasosiego y tranquilidad. Se configuró como un espacio donde los cuerpos pasean sin sospecha y sin rumbo.

El nuevo contexto social y urbanístico estaba marcado por un crecimiento de la población en las grandes ciudades, lo que provocaba el encuentro constante entre desconocidos. Este crecimiento poblacional y los nuevos usos del espacio público facilitaron que el encuentro sexual se pudiese articular sin grandes dificultades aparentes en estos escenarios que la propia ciudad ofrecía a sus ciudadanos. No obstante, no porque la mayor parte de estos enclaves para el sexo anónimo se ubiquen en la ciudad deberíamos creer que son lugares exclusivos de las grandes ciudades; áreas de descanso de autopistas, bosques en zonas rurales o playas alejadas también son espacios propios de este tipo de actividades. Ahora bien, tal y como señala Bech (1987), las redes de solidaridad y el surgimiento de la subcultura gay que hoy conocemos sí que debemos situarlas en la gran ciudad.

A pesar de las oportunidades de experimentación que brindaba la ciudad, esta no dejó de ser objeto de críticas y recelo precisamente por considerarse como el lugar que acoge las mayores perversiones. En el Estado español diferentes ensayistas mostraron su preocupación por los encuentros sexuales entre 
hombres en espacios públicos desde finales del siglo XIX. Consideraban que la nueva vida urbana era la responsable de la pérdida de valores tradiciones y de la depravación moral (Bernaldo de Quirós 1901, Carlavilla 1956, Max-Bembo 1912, Rodríguez-Solís 1891, Sabater 1961 y Suarez 1983). Es por ello por lo que, a pesar de que se daban las circunstancia para los encuentros anónimos, estos rápidamente se convirtieron en objeto de sanción y supervisión, lo que obligó a quienes participaban en esta actividad a buscar las estrategias que permitiesen cumplir el fin último del encuentro sin arriesgarse a ser sancionados, descubiertos o detenidos.

En las dos últimas décadas la creación de escenarios de ocio dirigidos a la población gay, así como la expansión del uso de las nuevas tecnologías con finalidades relacionadas con el encuentro sexual han provocado que muchos hombres no recurran a las zonas de cruising como lugares para el encuentro sexual y busquen sus parejas en otros espacios tales como bares, saunas, cruceros o páginas de contactos. Este tipo de locales y servicios se dirigen fundamentalmente a población que se identifica como gay, mientras que las zonas de cruising no se vinculan a ninguna identidad sexual y permanecen en la penumbra de la aceptabilidad social y su correspondiente sospecha asociada; por lo tanto, el acceso al sexo en las zonas de cruising todavía requiere de una estrategia que permita garantizar el anonimato de quienes participan en esta actividad.

\section{Narrativas y discursos}

La práctica del sexo anónimo, como cualquier otro hecho social, puede contar con diferentes narraciones en función del emisor del mensaje. Los discursos médicos y las instituciones de salud pública, por ejemplo, parten normalmente de una posición de atención a la salud sexual y de prevención de enfermedades de transmisión sexual, centran su atención en un aspecto concreto de la interacción que promueve únicamente una mirada sanitaria (Fernández-Dávila 2009, Flowers 1999, Keogh 2000 y Villaamil 2008). Desde las narrativas policiales, la práctica del cruising es una cuestión de seguridad ciudadana. La Asociación de policías gays y lesbianas (Gaylespol) ha publicado, algunas recomendaciones para los participantes de la práctica del cruising en las que orienta a los usuarios con algunos consejos de seguridad, entre ellos se puede destacar el siguiente:

"Si es tu primera visita, ve acompañado con alguien de confianza que te pueda guiar. No te precipites. Haz una primera vuelta, detecta posibles peligros y busca un lugar seguro para mantener sexo con quién te puedas encontrar. El lugar que escojas ha de tener una salida rápida en caso de padecer un ataque o acto delictivo. Evita cuevas, barrancos, y espacios donde puede que no te oigan en caso de pedir ayuda. Si antes de estar en una zona de noche, ya la has conocido de día, te ayudará a hacerte una idea mejor y controlar mejor los espacios" (Gaylespol s. f.).

Desde las pláticas políticas de las administraciones locales se oculta esta realidad para evitar los posibles conflictos vecinales $\mathrm{y}$, cuando pasa a formar parte del debate mediático, normalmente, se presenta como una problema de escándalo público y se intentan poner todos los medios que impidan su desarrollo. Este es el caso, por ejemplo, de Benalmádena (Málaga), o de Badalona (Barcelona), donde el alcalde, Xavier Albiol no tuvo reparos en hacer público en el 2012 su intención de acabar con la práctica del cruising en la playa de la Mora advirtiendo que "se acabará porque actuaremos de forma contundente" mediante una fuerte "presión contra los viciosos" (La Vanguardia, 9 de agosto de 2012).

También existen algunos escritores homosexuales que se muestran en contra de este tipo de actividades porque entienden que se trata de un escenario para la propagación del VIH (Rotello 1997 y Signorile 1997). No obstante, cabe señalar que otros autores consideran que difícilmente se puede evaluar si se producen más prácticas de riesgo en los lugares de sexo anónimo que en el espacio sexual doméstico (Clatts 1999). En cualquier caso, las zonas de cruising han sido tildadas como lugares sexuales despersonalizados y peligrosos, como si en ellos se llevase a cabo un sexo no social, cuando en realidad, el sexo, en cualquiera de sus formas es, sobre todo, social.

Algunos homosexuales también consideran que la práctica de cruising representa una promiscuidad y unos excesos que dañan la imagen del colectivo homosexual y por lo tanto dificultan la integración plena de los gays. 
"Que la sociedad identifique a los gays con tíos que van a lugares apartados a darse por el culo con unos y con otros sin preguntarse ni siquiera el nombre, ¿crees que no perjudica nuestra imagen de cara a la sociedad? ¿Crees precisamente que eso no le da argumentos a la extrema derecha? Mi juicio de valor no es nada subjetivo. De hecho, es muy objetivo. Cada uno es libre de hacer con su cuerpo lo que quiera, pero aquí nadie está exento de crítica. No os pongáis hipersensibles, porque no sois intocables, ¡eh! Lo creo personalmente, y siento mucho al que le ofenda, que esta práctica, que yo no comparto, no va asociada inherentemente a la homosexualidad, y yo no tengo porque defenderla. Esto no tiene nada que ver con la homofobia. Llamadle "cruisingfobia'" (comentario de un lector en el foro de la página web de noticias LGTB http://www.dosmanzanas.com).

Por el contrario, otros homosexuales valoran positivamente este tipo de escenarios y reconocen las posibilidades que ofrece un lugar para el sexo anónimo y gratuito en espacios públicos. "A mí lo que me gusta es el plan ese cachondo en el que va la peña. Eso de hacerse pajas delante tuyo", defendía un usuario de la zona de cruising de Montjuïc. Otro de los participantes destacaba que lo que le gustaba era el entorno en el que se llevaba a cabo y la tranquilidad con la que podían encontrar otras parejas sexuales.

La lista de oradores todavía podría aumentar más si pensásemos en los hombres heterosexuales que fantasean con lugares similares para el gozo sexual con mujeres, vecinos que viven próximos a este tipo de espacios, entidades católicas, asociaciones de defensa de los derechos de las personas LGTB, etc. Son innumerables los posibles discursos entorno a la práctica del sexo anónimo. Sin embargo, en raras ocasiones son los propios protagonistas de esta actividad quienes construyen sus narrativas, cuentan sus historias y elaboran un pasado. Las narrativas del cruising son narrativas que se han configurado desde el pensamiento hegemónico, desde la alteridad, el desprecio y la humillación. Este hecho hace difícil que los propios participante escapen del reconocimiento abyecto y articulen discursos públicos positivos de su experiencia. Resulta complicado reconocerse en aquello que constituye como un hecho vergonzante (Goffman 1963), verse entrometido en lo que no se debe hacer. ¿Cómo narrar lo que no se puede explicar?, ¿quiénes son los historiadores de la práctica del sexo anónimo?, ¿cuáles son los registros, las fuentes a consultar?, ¿las fichas policiales de detenciones?, ¿cómo se registra el deseo y las fantasías en una ficha policial?, ¿cómo se transmite el conocimiento adquirido en los parques de generación en generación? Es francamente complicado hacer una historia de una práctica secreta, subalterna, no generadora de identidad, sin referentes ni portavoces, una práctica que los sujetos borran de su historia social y solo se reserva para el recuerdo individual, a veces entrañable, pero nada más que recuerdo. Las narrativas del cruising son narrativas polimorfas donde cada sujeto encuentra un sentido a lo que allí sucede. Son al mismo tiempo historias de control y liberación, de supervivencia, de deseo y de perversión. Se trata de narrativas en las que se confunde lo sucedido con lo imaginado. "Aquí todos lo hemos pasado muy bien, mamando y follando con todo quisqui", se jactaba un participante en Gavà. Cuando las circunstancias lo requieren, también son narrativas de la mentira que permiten inventar nombres, profesiones y procedencias. Narrativas del desprecio de los demás y de uno mismo. "Ya es bastante vergonzoso venir aquí, como para encima ir contándolo", aseguraba otro practicante. Se trata muchas veces de narrativas que relatan una trayectoria y una historia de cambios:

"Llevo años en esto, ya vengo del Toro Bravo. Antes el rollo estaba en las cañas del Prat, después nos vinimos al Toro Bravo que no tenía barrera y aquello era una pasada. Allí te hinchabas de todo. Luego empezaron a joder el Toro Bravo y nos fuimos al otro lado de la autopista -jAnda que no hemos trotado alli!-. Y desde que lo cerraron nos vinimos aquí. Como si yo fuera el presidente de la comunidad (risas)".

Al fin y al cabo, las narrativas de cruising son narrativas que difícilmente pueden narrar sus propios actores. No obstante, algunos relatos literarios hacen referencia explícita a la práctica del sexo anónimo y describen las bondades de los encuentros entre hombres haciendo fantasear al lector y trasladándole los sentimientos, deseos y sensaciones íntimas que se pueden vivir en este tipo de lugares. Hollinghurst (1988), Lemebel (1995), Moore (1992) Orton (1986), Roig (1977) o White (2001) son algunos de los autores que se han ocupado de este asunto. Así relata Lluís Fernández (1979) en El anarquista desnudo uno de los posibles encuentros sexuales:

"Apoyado sobre un tronco de eucaliptus, abierto de piernas, recibe las primeras cálidas caricias de una lengua desconocida que, sin lugar a dudas, ejecutará un trabajo de marquetería digno de premio. Chupar es una vieja función que devuelve a la infancia. Micky tiene el pene tan sensibilizado que en el momento de recibir la tibieza bucal, al sentir la lengua lamiendo los recovecos del glande, comienza a 
gruñir y moverse como un demonio y le invade tanta furia, que me parece imposible que pueda detenerle persona alguna. El mago sube y se hunde cada vez con más fuerza dentro de la garganta, intentando llegar más allá de la campanilla y perforar el velo del paladar. Por la manera en que le tiene cogido, la víctima inocente recibe las primeras sacudidas que acabaran en un enloquecedor "dentro, dentro" y una descarga de semen tan sofocadora como una bocanada de agua salada y de estrellas" (Fernández 1979: 74).

A pesar de la existencia de relatos de autoafirmación de la práctica del cruising de algunos escritores, sería necesario preguntarse por las razones que conducen a diferentes actores sociales a tomar una postura sancionadora con respecto a este tipo actividad. Un análisis de estas reacciones no solo contribuye a comprender la estructura del pensamiento homofóbico, sino que también nos puede ayudar a entender cuáles son las estrategias de los participantes que deciden inmiscuirse en la actividad del cruising para acceder a los placeres carnales y evitar, a su vez, ser criminalizados.

Existen tres razones fundamentales que se articulan para organizar la crítica al sexo anónimo entre hombres en espacios públicos. La primera de ellas está relacionada con el objeto de deseo, es decir, la condena a las relaciones sexuales entre personas del mismo sexo por el hecho de constituir una relación sexual no reproductiva ni enmarcada en la lógica del deseo dentro binomio hombre/mujer. La homofobia se presenta como un conjunto de ejercicios de supervisión y exclusión que tienen por finalidad contribuir a la gestión social del sexo basada en el paradigma la heterosexualidad. El estudio de la homofobia ha sido objeto de análisis de diferentes autores y autoras como Eve K. Sedgwick (1990), Didier Eribon (1999), Jeffrey Weeks (1985) o Gayle Rubin (1984) en el plano internacional, y Gerard Coll-Planas (2010), Oscar Guasch (1991), Gracia Trujillo (2009), Jose María Valcuende (2006), Paco Vidarte (2007) o Fernando Villaamil (2004), entre otros, en el ámbito estatal. La existencia previa de trabajos sobre homofobia permite dar paso a otros elementos que contribuyen notablemente a explicar esta actividad. La segunda razón por la que la práctica del cruising está devaluada tiene que ver con uno de los principios que regula las parejas sexualmente legítimas: la monogamia. La monogamia como principio rector organiza las experiencias sexuales y hace que quienes decidan quebrantar esta norma se vean obligados a recurrir a estrategias de ocultamiento. Por todos es sabido que ser infiel a la pareja es algo que no se debe hacer y mucho menos decir. La variedad de experiencias sexuales de los sujetos parece que pesa en su contra, como si experimentar y aprender sobre el sexo fuese un hecho negativo en sí mismo. Cuando en realidad, la experiencia favorece el conocimiento sobre uno mismo y el descubrimiento de nuevas posibilidades y destrezas que pueden llevar al sujeto a una mayor satisfacción y placer. El sexo anónimo representa por ello la ruptura con un modo de relación social en el que la pareja se presenta como una finalidad en sí misma (Esteban 2011). Por lo tanto, la práctica del cruising no está en el punto de mira solo por el objeto de deseo de los participantes, sino también por su ruptura con la lógica monógama y de la obligatoriedad del sexo con amor y en pareja.

La tercera razón tiene que ver con el espacio en el que se desarrolla el encuentro. Los espacios públicos no se presentan como lugares para el sexo, al menos para el sexo homosexual, ya que las celebraciones de la heterosexualidad son una constante en el panorama urbano (Langarita 2013a). Los usos que maricas y bolleras pueden hacer del espacio público se articulan a partir de la aceptación de la lógica heterosexual, la familia y a las dinámicas del binomio hombre/mujer. Las expresiones no-heterosexuales, una vez reconocidas, merecen ser llevadas a cabo en el espacio privado e íntimo, convertirse en no-visibles. Se puede aceptar la homosexualidad, pero para ello debe comprometerse con no irrumpir en el proyecto "heterosexualizante" que ocupa el espacio público, o al menos no subvertirlo (Langarita 2013b). Es por ello por lo que la práctica del cruising interpela el orden sexual en cuanto al escenario en el que se lleva a cabo, ya que se trata de una actividad que da un uso no previsto a un espacio construido para la reproducción social de la heterosexualidad. Parques, lavabos públicos o playas son objeto de resignificación sexual por parte de los usuarios que se involucran en la actividad del cruising.

\section{Estrategias para el gozo}

La persecución y las críticas no evitan que las zonas de cruising den acceso al gozo sexual a un buen número de hombres, los cuales han elaborado un conjunto de estrategias que permiten hacer frente a las ignominias que se vierten contra ellos. Estas estrategias se organizan a partir de la ley del silencio (Langarita 2013c) y la ritualización de la práctica del sexo anónimo. Los participantes ponen en marcha 
diferentes ejercicios formalizados y expresivos que arrastran una dimensión simbólica y conducen el ritual al acto sexual. Pero, además, es importante destacar que esta forma de ritual permite preservar la sensación de anonimato de quienes se involucran en la práctica, y por lo tanto evita, en buena medida, la sanción social personificada.

Como otros rituales, la práctica del sexo anónimo tiene sus propias normas y procesos que, por fugaces que puedan ser los encuentros, generan en un marco de institucionalización entre quienes interactúan en ellos. Los rituales hacen que se produzca una atención y emoción entre los participantes que proporciona una realidad temporalmente compartida (Collins 2005: 21). Cuentan con un conjunto de reglas propias que determinan cuando se inicia y acaba el ritual de intención. Es por ello por lo que Laud Humphreys (1970: 47) asegura que las normas son más importantes que las estrategias para ligar, ya que son la vía para establecer la protección de uno mismo y del grupo con el que se comparte la interacción ritual. Humphreys (1970) enumera seis normas principales para el buen funcionamiento del espacio de intercambio sexual anónimo. Su propuesta nos puede servir de referencia para introducirnos en el análisis del ritual de interacción en las zonas de cruising: evitar el intercambio de datos personales, ¡Cuidado con los chaperos! (a veces quien no lo parece lo es), nunca forzar la interacción, no criticar a las otras parejas sexuales, no romper los acuerdos entre el grupo, permanecer en silencio durante la actividad del cruising.

Las personas deben ser capaces de adoptar las estrategias más adecuadas que conduzcan a la interacción sexual. Conocer las normas de funcionamiento es básico para participar satisfactoriamente en el ritual, de hecho aquellos hombres que no las respetan, rápidamente quedan excluidos de la interacción y tienen pocas garantías de éxito y acceso al sexo (Tewksbury 1996). Los participantes se sienten molestos cuando otras personas intentan ligar saltándose la norma del silencio o acechan demasiado rápido sin respetar el proceso del acuerdo (Langarita 2013c). De hecho, las personas que utilizan estas tácticas, llamémoslas de "urgencia sexual" por la rapidez que denotan en la demanda del sexo, no suelen ser bien acogidas por los participantes y, en muchas ocasiones, quienes recurren a ellas acaban saliendo de las zonas de cruising sin haber podido realizar ningún contacto sexual. Oscar Guasch asegura, en este sentido, que "cuando un varón suprime el cortejo e inicia el contacto (verbal o corporal) directamente es posible que sea rechazado porque no se ajusta al ritual" (Guasch 1991: 126). La ruptura de la norma también puede producirse por agentes externos que no forman parte de la interacción, pero, en estos casos, rápidamente son aislados o mantenidos al margen de las negociaciones sexuales. Este es el caso, por ejemplo, de turistas que se meten entre los arbustos del parque de Montjuïc para liberar los apretones de sus vejigas, o de la llegada de vecinos que se introducen en el parque para pasear a sus perros.

La dimensión simbólica de la práctica del cruising adquiere significado en la medida en la que es compartida por un grupo que la interpreta y la respeta. El sujeto que se inicia en la interacción espera que el resto de los hombres que merodean por el parque sepan interpretar sus intenciones para así poder conseguir el fin último del acceso al gozo sexual.

Las normas que ordenan la práctica del sexo anónimo cuentan con una gran eficacia, ya que son capaces de conducir a los individuos a la satisfacción de sus deseos sexuales sin la necesidad de arriesgar otros elementos personales o sociales. Permiten mantener el anonimato de los participantes y así evitar su descubrimiento. Para poder participar en la práctica del sexo anónimo es necesario, en primer lugar, realizar un ejercicio de aprendizaje tal y como se hace en cualquier actividad ritual (Durkheim 1912, Goffman 1967 y Segalen 1998). El aprendizaje consiste no solo en averiguar cuáles son los símbolos y sus significados válidos para cada ritual, sino que también reconocer el orden y usos de estos símbolos. La adquisición del nuevo saber se lleva a cabo, fundamentalmente, a través de la observación de los iniciados. No existen sesiones de preparación para el sexo anónimo tal y como sucede con otros rituales, no hay maestros que acompañen y guíen a los practicantes. Los nuevos usuarios observan el comportamiento de los hombres que circulan por la zona de ligue y se lanzan hacia la actividad gracias a una especie de pedagogía desviada que enseña sin palabras el proceso ritual a los recién llegados, los cuales rápidamente descubren las pautas de comportamiento, las normas a seguir y las vías para garantizar el éxito del ritual. Los símbolos que articulan la práctica del cruising son simples pero eficaces, permiten el acceso a la interacción, garantizan el anonimato de los interactuantes y conducen al acto sexual sin grandes dificultades. Por lo tanto el acceso al sexo requiere tanto de un espacio de socialización como de una estructura social y simbólica que se encargue de organizar el deseo (Green 2008: 28). De manera que la práctica del cruising no se puede reducir únicamente a un acto comunicativo, sino que, a pesar de que el lenguaje toma un papel fundamental en su desarrollo, el ritual también se produce a sí mismo en tanto que práctica social con 
diferentes actividades implícitas que requieren de la experiencia y la relación social (Bloch 2012).

Llegados a este punto es necesario preguntarse a cerca de cuáles son los símbolos que contribuyen a la ordenación de la práctica del sexo anónimo entre hombres. Víctor Turner (1980: 35) hace una distinción entre símbolos dominantes y símbolos instrumentales, en la que los primeros son relativamente fijos tanto en la estructura cultural como en la sociedad, mientras que los segundos tienen unos fines específicos dentro de un ritual concreto. Siguiendo esta propuesta podríamos pensar que los símbolos dominantes en las zonas de cruising están caracterizados por una organización social de la sexualidad basada en el paradigma de la heterosexualidad. La heterosexualidad deviene en algo más que una práctica sexual, consiste más bien en un modo de organizar la vida en sociedad, lo que la convierte en uno de los símbolos dominantes de nuestra sociedad. La posición social de heterosexual ofrece algunos privilegios que están fundamentalmente relacionados con el acceso al poder y a los recursos en tanto que sujetos heterosexuales (Bourdieu 1998 y Wittig 1992). En este sentido, Rubin (1984) defiende la existencia de una jerarquía sexual, en la que la heterosexualidad ocupa el lugar de mayor aceptación social.

Otro de los símbolos dominantes está enmarcado por el hecho social de la homofobia, a partir del cual se desprenden diferentes estrategias de criminalización y sanción para aquellos sujetos que no se adscriben al mandato sexual. Es decir, la práctica del cruising es una actividad en la que los participantes entran en negociación con el modelo social homófobo que organiza la sociedad occidental. En este sentido, quienes asisten a las zonas de cruising, así como el conjunto de personas que desean a otras personas de su mismo sexo, también cuentan con un marco de referencias simbólicas compartidas por el conjunto de la sociedad y que, en muchas ocasiones, pasan por la representación de la homosexualidad como un hecho indeseable y digno de ocultamiento.

El tercero de los símbolos dominantes que contextualizan la práctica del cruising es el de la monogamia, el cual acota la actividad sexual a la relación de pareja. Este principio presenta el sexo como una actividad vinculada al amor exclusivo y no como un instrumento para el gozo corporal.

Sin embargo, más que profundizar en la idiosincrasia de los símbolos dominantes, se tratará de analizar las características y ordenación de los símbolos instrumentales, es decir, de aquellos símbolos que tienen un fin específico dentro de este ritual. Mary Douglas (1970) considera que el ritual necesita de un lenguaje eficaz que permita actuar en la realidad social. En las zonas de cruising, este lenguaje se caracteriza por la ausencia de palabras, lo que provoca que los símbolos comunicativos se articulen fundamentalmente a partir de diferentes ejercicios corporales que se convierten en básicos para la comprensión y participación en esta actividad (Langarita 2013c). Gracias a esa normativización las acciones comunicativas permiten generar un entramado simbólico que conduce hacia el acto sexual entre los participantes, es decir, el conjunto de símbolos corporales que ordenan la actividad del cruising son la pieza clave para la eficacia del ritual que se representa mediante el acceso al sexo. El cuerpo se convierte así en un artefacto simbólico pleno en las zonas de cruising (Langarita 2013c). Los símbolos que se desprenden del cuerpo los podríamos clasificar en dos grandes bloques. En primer lugar, estarían los símbolos del cuerpo sustanciales, que refieren a aquellos símbolos que el participante transmite independientemente de su actitud y su comportamiento. Se trata de símbolos inherentes a su corporalidad y que el sujeto difícilmente puede manipular, pero que comportan un elemento fundamental para la interacción. Entre ellos estaría la edad, la etnia, la capacidad muscular o flacidez, la obesidad o delgadez, el olor o el vello corporal, entre otros. Los símbolos del cuerpo sustanciales se significan normalmente en las zonas de cruising a partir de las mismas atribuciones y valores que los de la sociedad madre donde se desarrolla el ritual. De esta manera, el éxito de la interacción se vincula al significado de los atributos de los que se dispone. No obstante, cabe señalar que no toda la escena gay comparte los mismos valores eróticos. De hecho, algunos elementos que en principio no resultarían atractivos en la sociedad madre ni en buena parte de la subcultura gay, sí que lo son para algunas subculturas homosexuales, como es el caso del vello corporal, por ejemplo.

En segundo lugar, estarían los símbolos del cuerpo manipulables. Estos son utilizados conscientemente por los participantes para conducir el acto ritual. En el caso de la práctica del cruising, la finalidad de los encuentros no requiere de un sistema complejo de comunicación corporal, con pocos elementos se resuelven las estrategias comunicativas. Se pueden distinguir tres símbolos básicos que en principio cuentan con momentos propios para cada uno de ellos, pero que, sin embargo, en una gran variedad de situaciones se utilizan de manera simultánea para intensificar la relación: el mantenimiento de la mirada; la demostración genital y su uso público; la forma de caminar, desplazamientos por la zona de cruising y 
posturas corporales. Richard Tewksbury (1996) sugiere, por su parte, que existen cuatro modos de comunicación no verbal en la zona de cruising: contacto ocular, persecución, exposición y contacto corporal.

En cualquier caso, la mirada ha sido desde hace tiempo una herramienta habitual en el ligue callejero, en parques y en otras zonas de intercambio sexual anónimo. Así lo describe un participante que vivió la persecución franquista a los homosexuales en Barcelona:

“¿Que cómo se ligaba? Pues en la calle, veías una mirada y te volvías, te ponías a mirar un escaparate, y el otro tío se ponía a mirar otro, y te mirabas, y poco a poco te ibas acercando y poniéndote a su lado, nos seguíamos de un sitio a otro y si tenías sitio pues te ibas a tu casa, o a su casa, algunos alquilaban habitaciones. Yo no alquilé nunca, pero sí que había gente que lo hacía. Y si no había nada, pues te ibas a un sitio oscuro y escondido y allí lo hacías".

En las zonas de cruising, la mirada se utiliza para determinar el interés sexual en otros hombres. Se trata de una mirada descarada, que no se retira cuando el sujeto es descubierto, sino que se mantiene y se intensifica para buscar complicidad, no debería desprender temor sino seguridad y deseo. La mirada en las zonas de cruising no es un acto de observación, se trata de un acto de comunicación que sirve para reconocer el interés en los otros participantes. Así lo explicaba un usuario: "El otro día un tío cruzó del otro lado del bosque y se me quedó mirando. Yo iba a la playa, no venía a ligar, pero se metió por aquí. Y dije, pues voy a probar, me gustó y él se quedó mirando. Y luego lo pillé aquí dentro y ipara delante!”. Otro participante aseguraba que "Si un tío me gusta yo lo miro y si él me mira quiere decir que está interesado en mí, si voltea la mirada quiere decir que no".

La mirada es, por lo tanto, una estrategia que requiere de la complicidad de las partes y que sirve tanto para demostrar interés como para descartar la disposición al sexo (Tewksbury 1996). Cuando un individuo desea rechazar una interacción que se inicia con una mirada mantenida de otro hombre, simplemente debe desatenderla para aclarar su abulia hacia la interacción. No obstante, en algunas ocasiones, el desprecio al inicio de la negociación sexual también se acompaña con otros signos, como marcharse del espacio hacia otra zona de la misma área de cruising, o caminar unos pasos hacia atrás de la persona que había iniciado la interacción.

El mantenimiento de la mirada es el primer elemento comunicativo para iniciar la interacción sexual. Pero esta mirada no es una mirada estática y fija a lo largo de la negociación, va cambiado y adaptándose al proceso de acercamiento a los otros participantes. Una vez los interactuantes mantienen su mirada el uno en el otro, esta cambia de forma y toma un matiz más lascivo que se acompaña, normalmente, con otros gestos faciales y corporales, como pequeños juegos de demostración de deseo simulando besos en los que simplemente se arquean los labios, juegos con la lengua ante los ojos de la pareja sexual, o se inicia la manipulación de los propios genitales.

La muestra y manipulación de los genitales se puede usar en diferentes momentos de la interacción ritual. De hecho, algunos de los usuarios muestran sus genitales directamente al conjunto de hombres que pasan por la zona de cruising sin iniciar necesariamente el ritual de interacción, simplemente los ponen a disposición de cualquiera de los participantes que quisiera acceder a ellos. En este caso, los hombres se saltarían la fase previa de mantenimiento de mirada, aunque, como he señalado, no por ello cuentan con mayores garantías de éxito. De alguna manera, la polla se presenta como un atributo al servicio del gozo de otros hombres y de uno mismo, facilitando así el acceso a la negociación y aclarando la disposición al sexo de manera determinante. (Con el uso del término "polla" pretendo destacar el significado cultural de este concepto).

La mayor parte de los hombres recurren a la estimulación genital una vez han iniciado la negociación. Cuando la pareja con la se ha comenzado la interacción visual mantiene su interés, los hombres comienzan a focalizar la atención en sus genitales mediante tocamientos con la mano y un acompañamiento de la mirada que se dirige directamente a la polla. Estos pequeños masajes también pueden ir asociados a tocamientos del propio pecho; en cualquier caso, el mensaje siempre está muy genitalizado. En algunas ocasiones estos masajes se realizan dentro de los pantalones, pero siempre intentando denotar erección a la futura pareja sexual. En otras, los individuos sacan su polla erecta para que la pareja sexual la pueda ver directamente. La erección es una señal fundamental en las primeras fases del juego sexual. Todos los hombres deben tener la polla erecta en este momento de la interacción. La polla es un elemento imprescindible y necesario para el cruising, sin pollas no hay cruising. 
Por el contrario, los participantes no van mostrando su culo para que el resto de los hombres puedan sentirse atraídos por él, nadie suele gesticular deseo entorno a su culo en los procesos de negociación que conducen al sexo. A pesar de que se trata de una zona corporal muy sexuada en el entorno homosexual, apenas se producen interacciones sexuales que conlleven penetración anal en las zonas de cruising. No obstante, cabe la preguntase a cerca de cómo significa el culo. Con frecuencia, el culo es percibido como un elemento instrumental para el placer, pero se presenta como si se tratase de un orificio que puede desvincularse del cuerpo, que no corresponde al sujeto que lo porta. Mientras que la polla se convierte en un elemento social de más o menos libre disposición y que los hombres muestran a los otros participantes para despertar interés sexual, el culo es esencialmente privado, pertenece a uno mismo y no debe trascender de la esfera íntima que lo aloja (Hocquenghem 1972), como si la privacidad del culo fuese la prueba definitiva de la hombría (Saez 2010). Pero el debate va más allá ya que, tal y como presentan Javier Saez y Sejo Carrascosa, "el problema que planta el culo es que todo el mundo tiene uno. Eso coloca a los hombres y mujeres en una cercanía demasiado peligrosa en el sentido en que convierte a ambos cuerpos en penetrables" (2010: 119). Frente a la polla, que se presenta como el órgano incontrolable que solo los hombres pueden poseer y se ven obligados a satisfacer de cualquier forma, el culo, en tanto que órgano penetrable, pone en cuestión la división sexual del género, ya que es la prueba de que todas las personas puedan ser penetradas. En este sentido Mary Douglas sostiene que "solo exagerando la diferencia entre el adentro y afuera, encima y debajo, macho y hembra, a favor y en contra se crea la apariencia de orden" (Douglas 1966: 22). El culo no tiene género, pero la polla es eminentemente masculina y por ello se hace más visible. Una mayor presencia del culo como objeto de deseo y placer corporal podría quebrantar las lógicas del binarismo sexual.

Continuando con el proceso ritual, una vez los practicantes han llegado al punto de mirada mutua acompañada de estimulación genital individual, acostumbran a ir acercándose poco a poco con el mantenimiento de la mirada hasta estar el uno frente al otro. En este momento comienzan a estimular, sin mediar palabra, los genitales de su pareja sexual, lo que suele conducir al sexo oral y a la masturbación individual o mutua. Así describía este proceso en mi diario de campo los primeros días de acceso a la zona de cruising de Gavà:

"Poco a poco, uno de ellos se me fue acercando. Este guardó la distancia durante mucho tiempo, me rodeaba y se puso a mi lado, primero a cuatro o cinco metros, me miraba, luego me fui acercando yo y me quedé a tres metros, nos fuimos arrimando el uno al otro hasta que nos quedamos a dos metros más o menos y nos pusimos uno frente al otro con esa distancia. Él me miraba, se tocaba el pecho y los pezones. Me estaba haciendo constantes señales de deseo sexual, yo permanecía quieto observándolo. Finalmente, se acercó y me tocó el pecho metiendo los dedos entre los huecos de mi camisa, me continuó tocando y enseguida puso su mano sobre mi polla, me la sacó y comenzó a pajearme" (extraído de mi diario de campo).

La técnica de caminar se inicia habitualmente después de pasar al lado de otro hombre o haber mantenido una breve mirada mutua. Uno de ellos (sujeto A) comienza a seguir al otro (sujeto B) mientras pasea por la zona de encuentro. Es decir, mientras el sujeto $B$ va caminando, el sujeto $A$ comienza a caminar detrás de él a una distancia aproximada de tres a cinco metros, lo que provoca normalmente que el sujeto B reduzca el ritmo de su paso en caso de que esté dispuesto a iniciar la negociación. Si el sujeto B no quiere llevar a cabo el proceso de negociación, suele acelerar para trasmitir al sujeto A su desinterés. Cuando el sujeto $A$ entiende este mensaje, abandona la persecución y continúa en búsqueda de otra pareja sexual diferente. En el caso en que el sujeto $B$ acepte la entrada al proceso de negociación, acostumbra a disminuir su ritmo al caminar, normalmente continúa andando unos segundos hasta que se asegura que le están siguiendo a él. Es en ese momento cuando busca un sitio donde detenerse para permitir que el sujeto A, que le está persiguiendo, le adelante. Este adelantamiento se produce a distancias muy cortas entre los dos participantes, en ocasiones incluso se produce un roce entre ambos cuerpos y algunos tocamientos, de manera que los dos se puedan ver de cerca e ir cerrando la negociación. Entonces, el sujeto A, después de adelantar, si desea continuar con la negociación también se detiene, esta vez a tres o cuatro metros del sujeto $B$ y comienza a iniciar la negociación a partir de la técnica de la mirada mantenida que se acompaña de juegos de provocación hasta que, finalmente, acceden al acuerdo y buscan un lugar donde llevar a cabo el acto sexual. Cabe señalar que este proceso de adelantamiento puede repetirse en varias ocasiones hasta cerrar el pacto sexual, normalmente, tocándose las pollas. 
Existen espacios donde se produce una negociación singular. En el caso de la zona de cruising de Montjuïc, podemos analizar las reuniones que se crean debajo de un puente. Se trata de un espacio oscuro, poco transitado y que permite resguardarse del frío, la lluvia o la visibilidad de los viandantes. En este punto los participantes se reúnen en silencio formando un círculo en el que todos pueden verse. Si se desea iniciar la negociación con uno de ellos, los participantes suelen acercarse recurriendo a la técnica de la mirada mantenida con las sucesivas provocaciones asociadas y sin dejar de mirar al hombre elegido para la interacción. El paso siguiente sería el sello del acuerdo sexual, aunque este acto no suele darse debajo del puente si se quiere una relación sexual de solo dos personas. De manera que los usuarios, una vez han iniciado el proceso de negociación y han obtenido una respuesta satisfactoria con la mirada, acostumbran a salir y dirigirse hacia una zona más aislada donde tener sexo entre ellos dos solos. No obstante, en algunas ocasiones, las prácticas sexuales se pueden realizar directamente debajo del puente, pero con la intención de que se forme una interacción grupal en la que diversas personas puedan participar y se extienda el juego sexual a cuatro, cinco o más personas.

Conviene aclarar que el rechazo a la interacción sexual no es una ruptura del ritual sino que, por el contrario, está contemplado como una posibilidad más. La negación para la interacción sexual no supone una humillación para los participantes, simplemente conduce a nuevos intentos (Frankis 2009: 874). En una ocasión, mientras hacía trabajo de campo en Montjuïc, pasó un chico magrebí de unos treinta años aproximadamente muy cerca del árbol en el que estaba apoyado, me miró y lo miré, mantuvo su mirada, pero después miró también a otro chico que estaba apoyado en el árbol de al lado. Se fue por el sendero y a los pocos pasos regresó. Volvió a pasar por delante de nosotros hasta tres veces, cada vez más cerca. Se marchó nuevamente por otro sendero, pero antes de entrar en él se giró para mirarnos. El chico que tenía a mi lado fue tras él, vi cómo se encontraron entre unos arbustos, pero enseguida el muchacho magrebi volvió hacia el sendero principal, había rehusado el encuentro. Pasó de nuevo a mi lado buscando una complicidad a la que no correspondí. El otro chico se apoyó en el árbol en el que estaba antes de marchar de persecución y la situación volvió a la normalidad.

Es importante dar cuenta de que el ritual de interacción sexual no se inicia únicamente en la fase de negociación y termina con el orgasmo. El ritual de interacción sexual se inicia en el momento en que los participantes se introducen en la zona de cruising y comienzan la selección y búsqueda de parejas sexuales, y finaliza en el momento en que se abandona el espacio. Incluso sugiero que, en el caso de Montjuïc, el ritual de interacción sexual se extiende a las calles adyacentes del parque, donde ya es posible ver a hombres que se dirigen hacia la zona de cruising. En el caso de Sitges, también se inician los primeros contactos visuales entre los bañistas de la playa cercana al bosque o en los bares nocturnos.

Resulta imposible hacer una descripción integra del ritual de interacción que recoja todas las posibles significaciones, ya que a pesar de la existencia de unos símbolos compartidos, las excepcionalidades de cada negociación la hacen particular. La jornada en las zonas de cruising puede ser muy variada en función del espacio, el tipo de usuarios presentes, las prisas de cada participante, la lluvia, u otros factores añadidos que condicionan la interacción. Sin embargo, para presentar una mejor perspectiva del proceso ritual es interesante aventurarse con una descripción de la interacción que parta de la propia experiencia en el campo y permita entender el proceso de negociación y pacto sexual:

"Se notaba que había comenzado el tiempo veraniego porque anochecía más tarde y la gente iba al parque a partir de las 20:30. Cuando llegué, ya era prácticamente de noche, pero todavía se podía ver con luz natural. Los arbustos habían crecido bastante con respecto al invierno y, por lo tanto, había más sitios para esconderse. También había más gente que la que hay normalmente. Alrededor de veinticinco hombres merodeaban por los senderos mirándose los unos a los otros e intentando buscar complicidades. En general, todos eran mayores de treinta y cinco años, incluso podían verse algunos de sesenta o probablemente más. La mayor parte de ellos parecían magrebíes y los más mayores, paquistaníes o bengalís. También había algunos catalanes y españoles de mediana edad.

Hice el paseo por el sendero principal, desde donde no pude observar a nadie manteniendo relaciones sexuales, aunque sí algunos hombres haciendo juegos de miradas. A mi llegada al final del sendero solo vi a una persona debajo del puente, hecho que me sorprendió, ya que durante mis visitas anteriores era habitual que debajo del puente se refugiasen bastantes hombres. Era un hombre mayor, que parecía que estaba solo esperando que alguien entrase allí. Continué entre los diferentes senderos de la parte superior, donde también había otros hombres con los que me crucé mientras caminaba, 
pero no había escenas visibles de sexo. No obstante, un par de ellos estaban en un proceso avanzado de la negociación. Volví nuevamente al sendero principal para a subir a la parte alta del parque. Cerca del puente había más hombres que cuando pasé por primera vez. Uno de ellos intentó iniciar el juego de negociación conmigo, me miró fijamente cuando pasé cerca de él, pero yo no respondí a su mirada y volví a la zona de la entrada donde me quedé un buen rato apoyado en un árbol viendo como nuevos participantes entraban a la zona de cruising. Después de un tiempo, un señor paquistaní se acercó para hablar conmigo. Me preguntó cómo me llamaba, le conteste y le devolví la pregunta, "Daniel", respondió. Sin embargo no lo creí, ya que su castellano denotaba un fuerte acento extranjero y estaba seguro de que Daniel no era un nombre habitual entre hombres paquistaníes de esa edad. Me preguntó a qué me dedicaba y dónde vivía. Todas estas preguntas me sorprendieron muchísimo, tuve la sensación de que estaban fuera de lugar. No era información importante que necesitara saber de mí para iniciar la negociación, así que no le he contesté. Algo de lo que posteriormente me arrepentí, ya que me hubiese abierto el camino para seguir con la conversación y obtener más información. Seguidamente me tocó el paquete, pero le dije que no buscaba nada. Me volvió a tocar el paquete un instante y se marchó.

Paseando de nuevo por un sendero me lo volví a encontrar. Trató de iniciar otra vez el acercamiento conmigo impidiéndome el paso e intentando darme un beso, algo extraño en la zona de cruising, pero, a pesar de que lo hizo de una forma divertida, no lo consiguió y yo continué por mi camino.

De nuevo pasé al lado del puente. El señor que había antes continuaba sentado solo. Me quedé mirando un rato para poder observar todos los movimientos desde la zona alta, aunque sin poder ver a nadie teniendo sexo. Un señor mayor, probablemente español, se me acercó, me tocó la polla por encima de los pantalones y se marchó. Yo seguí caminando, bajé al sendero principal, y desde allí me dirigí hacia otro sendero secundario que va por la parte baja y donde solía haber gente teniendo sexo. En esta ocasión, vi a dos personas masturbándose mutuamente. Se trataba de dos chicos jóvenes de los que no he podido identificar el origen debido a la oscuridad. También había una tercera persona con aspecto de ser de Europa del Este que estaba haciendo de voyeur. Cuando pase a su lado, este último trató de buscar el contacto con la mirada y luego con el cuerpo, pero yo pasé de largo y volví al sendero principal.

Nuevamente inicié un paseo por este sendero, donde vi a otras dos personas que se estaban pajeando mutuamente. Pero uno de ellos se marchó rápidamente cuando llegué, no sé por qué. En el puente, esta vez, había más gente. Había cuatro hombres y yo me quedé allí. Uno de ellos me hizo gestos con la cabeza para que le siguiese, lo hice, y se dirigió hacia fuera del parque. Como yo no quería salir, decidi cambiar de dirección, entonces me siguió él a mí. Nos colocamos enfrente y, sin mediar palabra, nos pusimos a masturbarnos mutuamente. Después me propuso que le mamase la polla, a lo que le contesté que no. Le propuse que lo hiciese él, pero tampoco quiso. Me ha insinuó que quería follarme y me negué. 'Entonces te haré correr de gusto', me advirtió. Me masturbo un rato hasta que me corrí. Se sacudió el semen de la mano y se limpió restregando su mano en la pared, yo me subí los pantalones y me marché de la zona de cruising" (extraído de mi diario de campo).

\section{Conclusiones}

La práctica del cruising es un acto ritual que va más allá de los formalismos e imprime ciertas obligaciones para quienes quieren acceder al sexo, o mejor dicho, para quienes quieran obtener los beneficios sexuales de esta interacción. Se trata de una actividad que no genera sentimiento de pertenencia, aunque sí vínculo, un vínculo temporal con todos aquellos hombres con los que se comparte el parque. El hecho de merodear por allí contribuye al intercambio de miradas y a la sugestión de expectativas. No obstante, este vínculo se rompe cuando los participantes salen de la zona de cruising, ya que en su vida social, trabajo o familia, las experiencias de sexo anónimo no suelen estar presentes, o al menos no son comentadas y compartidas. Por lo tanto, cada vez que los participantes se involucran en esta interacción no adquieren identidad social, tal y como sucede con otros rituales, pero, sin embargo, sí que re-evocan los símbolos que organizan la estructura social de las zonas de cruising y contribuyen a su mantenimiento.

Las personas que van a las zonas de cruising lo hacen, fundamentalmente, para sumarse a las experiencias placenteras del cuerpo tratando de esquivar las regulaciones sexuales que nuestra sociedad organiza a 
partir de los paradigmas de la heterosexualidad, la homofobia y la monogamia. Para adherirse a estos placeres, deben asumir las normas que operan y ordenan este tipo de espacios. Las zonas de cruising son una alternativa que permite acceder al gozo sexual con otros hombres sin ver comprometida la reputación de los participantes y, para ello, la ritualización de la actividad es un elemento clave que posibilita que los hombres puedan tener sexo con otros hombres de manera segura. Pero la noción de seguridad, en este caso, no responde tanto a cuestiones relativas a la delincuencia o a la prevención de enfermedades de transmisión sexual, como a no ver comprometidos otros aspectos de la vida social fuera del intercambio sexual anónimo, ya que este descubrimiento podría afectar seriamente a los usuarios a causa de su participación en este tipo de interacción. La ritualización de esta práctica es una herramienta indispensable para cumplir el objetivo de mantener el anonimato de los participantes. Generar un modo de relación normativizado a partir del ritual garantiza su cumplimiento, especialmente cuando la normativa es eficaz. Por lo tanto, el respeto del ritual de interacción en las zonas de cruising cumple dos de los objetivos de muchos de los participantes. Por un lado permite el acceso al sexo y, por otro, garantiza el anonimato de los participantes en un contexto de hostilidad social.

\section{Bibliografía}

Aldrich, Robert

2004 "Homosexuality and the City: an Historical Overview", Urban Studies, nº 41 (9): 1719-1727.

DOl: $10.1080 / 0042098042000243129$

Bech, Henning

1987 When men meet. Cambridge, The University of Chicago Press, 1997.

Bernaldo de Quirós, Constancio (y Jose María Llanas Aguilaniedo)

1901 La mala vida en Madrid: estudio psico-sociológico con dibujos y fotograbados del natural. Madrid, B. Rodríguez Serra.

Bloch, Maurice

2012 Anthropology and the Cognitive Challenge. Cambridge, Cambridge University Press.

Bourdieu, Pierre

1998 La dominación masculina. Barcelona, Anagrama, 2005.

Carlavilla, Mauricio

1956 Sodomitas. Homosexuales, políticos, científicos, criminales, espías, etc. Madrid, Nos.

Clatts, Michael C.

1999 "Ethnographic Observation of Men who Have Sex with Men in Public", en William Leap (ed.), Public Sex. Gay Space. Nueva York, Columbia University Press: 141-156.

Collins, Randall

2005 Cadenas rituales de interacción. Barcelona, Anthropos, 2009.

Coll-Planas, Gerard

2010 La voluntad y el deseo. La construcción social del género y la sexualidad: el caso de lesbianas, gays y trans. Barcelona y Madrid, Egales.

\section{Dos Manzanas}

s.f. 'El alcalde de Badalona, Xavier García Albiol, llama 'viciosos' a los practicantes de cruising y anuncia medidas policiales".

www.dosmanzanas.com/2012/08/el-alcalde-de-badalona-xavier-garcia-albiol-llama-viciosos-a-lospracticantes-de-cruising-y-anuncia-medidas-policiales.html 
Douglas, Mary

1966 Pureza y peligro. Un análisis de los conceptos de contaminación y tabú. Madrid, Siglo XXI, 1973. 1970 Símbolos naturales. Madrid, Alianza, 1978.

Durkheim, Émile

1912 Las formas elementales de la vida religiosa. Madrid, Akal, 1982.

Elias, Norbert

1977 El proceso de la civilización. Investigaciones sociogenéticas y psicogenéticas. México, Fondo de Cultura Económica, 1989.

Eribon, Didier

1999 Reflexiones sobre la cuestión gay. Barcelona, Anagrama, 2001.

Esteban, Mari Luz

2011 Crítica del pensamiento amoroso. Barcelona, Bellaterra.

Fernández-Dávila, Percy

2009 "Necesidades no-sexuales que motivan a un grupo de hombres que tienen sexo con hombres a involucrarse en prácticas sexuales de alto riesgo", Forum: Qualitative Social Research, n 10 (2), art. 21.

Fernández, Lluis

1979 El anarquista desnudo. Barcelona, Anagrama.

Flowers, Paul (y otros)

1999 "Constructing sexual health. Gay men and "risk" in the context of a public sex environment". Journal of Health Psychology, nº (4): 483- 495. DOl: 10.1177/135910539900400403

Frankis, Jamie S. (y Paul Flowers)

2009 “Public Sexual Cultures: A Systematic Review of Qualitative Research Investigating Men's Sexual Behaviors with Men in Public Spaces", Journal of Homosexuality, nº 56 (7): 861-893.

DOl: $10.1080 / 00918360903187846$

Gaylespol

s.f. Ligando al aire libre con seguridad. www.gaylespol.es/airelibre/

Goffman, Erving

1963 Estigma. La identidad deteriorada. Madrid, Amorrortu. 2003.

1967 Ritual de la interacción. Buenos Aires, Tiempo Contemporáneo, 1970.

Green, Adam Isaiah

2008 "The Social Organization of Desire: the Sexual Fields Approach", Sociological Theory, 26 (1): 25-

50. DOl: 10.1111/j.1467-9558.2008.00317.x

Guasch, Oscar

1991 La sociedad rosa. Barcelona, Anagrama.

Hahn, Pierre

1979 Nos ancêtres les pervers. La vie des homosexuels sous le second empire. París, Olivier Orban.

Hocquenghem, Guy

1972 El deseo homosexual. Barcelona, Melusina, 2009.

Hollinghurst, Allan

1988 La biblioteca de la piscina. Barcelona, Anagrama, 2006.

Humphreys, Laud

1970 Tearoom Trade. Impersonal Sex in Public Places. Nueva York, Enlarged, 1975. 
Keogh, Peter (y otros)

2000 "Anonymous sex among homosexually active men: implications for HV prevention", Venereology, $\mathrm{n}^{\circ} 13$ (4): 143-148.

Langarita, José Antonio

2013a "Ciudad, sexo y estigma. Una aproximación a la práctica del sexo anónimo entre hombres en espacios públicos", en José María Valcuende del Río (y otros) (coords.), Diversidad sexual en Iberoamérica. Sevilla, Aconcagua: 335-342.

2013b "Apropiaciones furtivas de espacios públicos: Intercambio sexual anónimo entre hombres en el entorno urbano", Quaderns-e de l'Institut Català d'Antropología (Barcelona), nº 18 (1): 99-113.

2013c "Sexo sin palabras. La función del silencio en el intercambio sexual anónimo entre hombres", Revista de Antropología Social (Madrid), n²2: 313-333. DOl: 10.5209/rev_RASO.2013.v22.43193

La Vanguardia

2012 "Albiol promete contundencia y presión policial contra el cruising y la prostitución de la playa de la Mora", La Vanguardia (Barcelona), 9 de agosto.

http://www.lavanguardia.com/local/20120809/54335822766/albiol-promete-contundencia-cruising-la-

mora.html

Lemebel, Pedro

1995 La esquina es mi corazón. México, Planeta, 2013.

Max-Bembo

1912 La mala vida en Barcelona. Anormalidad, miseria y vicio. Barcelona, Maucci.

Moore, Oscar

1992 Un asunto de vida y sexo. Barcelona, Tiempos Modernos.

Orton, Joe

1986 Diarios. España, Cabaret Voltaire, 2010.

Rodríguez-Solis, Enrique

1891 Historia de la prostitución en España y América. Madrid, Imprenta de Fernando Cao y Domingo del Val.

Roig, Antonio

1977 Todos los parques no son un paraíso. Barcelona, Planeta.

Rotello, Gabriel

1997 Sexual Ecology: AIDS and the Destiny of Gay Men. Nueva York, Dutton.

Rubin, Gayle

1984 "Reflexionando sobre el sexo: notas para una teoría radical de la sexualidad", en Carole S. Vance (comp.), Placer y peligro. Explorando la sexualidad femenina. Madrid, Talasa: 113-190.

Sabater Tomas, Antonio

1961 Gamberros, Homosexuales, Vagos y Maleantes. Barcelona, Hispano Europea.

Saez, Javier (y Sejo Carrascosa)

2010 Por el culo. Políticas anales. Madrid y Barcelona, Egales.

Sedgwick, Eve K.

1990 Epistemología del armario. Barcelona, Tempestad, 1998.

Segalen, Martin

1998 Ritos y rituales contemporáneos. Madrid, Alianza, 2005. 
1977 El declive del hombre público. Barcelona, Anagrama, 2011.

1994 Carne y piedra. El cuerpo y la ciudad en la civilización occidental. Madrid, Alianza, 2002.

Sibalis, Michael

1999 "Paris", en David Higg (ed.), Queer Sites: Gay Urban Histories Since 1600. Londres y Nueva York, Routledge: 10-37.

Signorile, Michelangelo

1997 Life Outside: The Signorile Report on Gay Men: Sex, Drugs, Muscles, and the Passages of Life. Nueva York, Harper-Collins.

Suarez, Vicente

1893 Conocimientos para la vida privada. La pederastia. Barcelona, Maurici.

Tewksbury, Richard

1996 "Cruising for Sex in Public Places: The Structure and Language of Men's Hidden, Erotic Worlds", Deviant Behavior, $\mathrm{n}^{\circ} 17$ (1): 1-19. DOl: 10.1080/01639625.1996.9968012

Turner, Víctor

1980 La selva de los símbolos. Madrid, Siglo XXI, 2005.

Trujillo, Gracia

2009 Deseo y resistencia. Treinta años de movilización lesbiana en el Estado español. Madrid y Barcelona, Egales.

Valcuende, José María

2006 "De la heterosexualidad a la ciudadanía", AlBR. Revista de Antropología Iberoamericana, n 1 (1): 225-242.

Vázquez García, Francisco (y Richard Cleminson)

2010 Los invisibles. Una historia de homosexualidad masculina en España 1850-1939. Granada, Comares.

Vidarte, Paco

2007 Ética marica. Proclamas libertarias para una militancia LGTBQ. Madrid y Barcelona, Egales.

Villaamil, Fernando

2004 La transformación de la identidad gay en España. Madrid, Catarata.

Villaamil, Fernando (y María Isabel Jociles)

2008 "Diferencias y desigualdades entre los HSH usuarios de locales comerciales de encuentro sexual: algunas contribuciones a las estrategias comunitarias de prevención del VIH", Etnográfica (Portugal), n 12 (2): 185-321.

Weeks, Jeffrey

1985 El malestar de la sexualidad. Madrid, Talasa, 1993.

White, Edmund

2001 The Flâneur. Londres, Bloomsbury Publishing.

Wittig, Monique

1992 El pensamiento heterosexual y otros ensayos. Madrid y Barcelona, Egales, 2006. 